\title{
Efficient Intercarrier Interference Mitigation for Pilot-Aided Channel Estimation in OFDM Mobile Systems
}

\author{
Ingmar Groh and Armin Dammann
}

\begin{abstract}
Motivated by the possibility of decreasing the intersymbol interference (ISI) which is due to large delays of a multipath mobile radio channel, orthogonal frequency division multiplexing (OFDM) became very popular. However, the timevariance of the mobile radio channel induces intercarrier interference (ICI) yielding substantial channel estimation errors and thereby tremendous transmission impairments. Contrary to previous algorithms which resort to a linearization of the time-variant channel, we combat the ICI using eigenspaces of time-domain covariance matrices defined by the autocorrelation function of the Doppler spread. We perform a basis expansion using Slepian sequences and determine the basis coefficients of the time-variant channel by channel estimates from previous OFDM symbols. Once we know these basis coefficients, we obtain the necessary time-variant channel estimation by the Slepian sequences. These time-variant channel estimates allow a symbol detection in frequency domain which eliminates the ICI almost completely. Simulation results investigate both the signal to interference ratio (SIR) and the bit error ratio (BER) of our new ICI mitigation methods and reveal the superiority compared to previous algorithms for ICI reduction.

Index Terms - orthogonal frequency division multiplexing, pilot-aided channel estimation, intercarrier interference, Slepian functions, basis expansion
\end{abstract}

\section{INTRODUCTION}

Orthogonal frequency division multiplexing (OFDM) allows to decrease the effect of intersymbol interference (ISI) due to a large delay spread and enables a simple subcarrier separation in the transmitter and receiver. For this reason, OFDM modulation enables very high data rates which is exploited in the digital video broadcasting (DVB) standard [1] for terrestrial (DVB-T) as well as handheld (DVB-H) reception. The high data rates are also important for fast short range communication in wireless high performance local area networks (HIPERLAN2) [2], [3]. However, the intercarrier interference (ICI) due to a non-vanishing Doppler spread needs to be taken into account as well. This Doppler induced ICI even increases if the bandwidth is divided by a larger number of subcarriers with a smaller subcarrier distance.

In order to decrease the ICI, the authors in [4], [5] resort to receiver diversity. All other previous ICI mitigation algorithms have in common the channel approximation by Taylor series [6], [7] or Lagrangian polynomials [8].

We present our new approach for the efficient ICI mitigation for pilot-aided OFDM channel estimation using the frame structure designed in [7] to show the advantages of our algorithms. In this paper, we follow the Fourier interpolation

I. Groh and A. Dammann are with the German Aerospace Center (DLR), Institute for Communications and Navigation, 82234 Wessling, Germany. E-mail \{Ingmar. Groh, Armin.Dammann\}@dlr.de.
[7] approach to get channel estimates for the data carriers from those for the pilot carriers. This interpolation method requires the total number of pilot tones for each OFDM symbol to exceed the guard interval length at least by one [9]. The arrangement of pilot and data symbols has equally spaced pilots in frequency direction [7]. Therefore, the exemplary OFDM frame corresponds to the HIPERLAN2 structure [2], [3] and to the continual pilot carriers (CPCs) for DVB-T and DVB-H OFDM systems.

Our novel ICI mitigation approach approximates the timevariant channel using the eigenmodes of time-domain covariance matrices. These eigenspaces are termed Slepian subspaces [10], [11] and are the optimum choice to minimize the mean square error (MSE) between a time-variant channel vector and its reduced-rank approximation. The Slepian subspaces also avoid further deficiencies compared to the Fourier basis like the Gibbs phenomenon [10], [11]. Clearly, the time-domain covariance matrices based on those OFDM symbols used for ICI mitigation depend on the sampling time and maximum Doppler frequency which we assume to be known at the receiver. The basic idea behind our ICI mitigation is to determine the coefficients of the time-variant channel with respect to the Slepian sequences by channel estimates from previous OFDM symbols. A determination requires at least as much pilot-aided channel estimates from previous OFDM symbols than basis coefficients of the Slepian sequences. The knowledge of these coefficients leads to the required reduced-rank time-variant channel estimation by the Slepian sequences. This result enables a symbol detection on the data carriers in frequency domain which reduces the ICI substantially. These steps resemble the Taylor series based ICI mitigation in [7] which uses channel estimates from adjacent OFDM symbols to perform an ICI reduced data detection in frequency domain. However, the results for high mobility OFDM systems show the advantages of our new ICI mitigation due to a better time-variant channel estimation.

Notation : Column vectors and matrices are denoted by small and capital bold letters, and the discrimination between deterministic (e.g. $\boldsymbol{y} \in \mathbb{C}^{N}$ ) and random quantities (e.g. $\boldsymbol{y} \in$ $\left.\mathbb{C}^{N}\right)$ is done by serifless font. We discriminate all functionals (e.g. $\mathrm{g}(t))$ using operator font. 'E $[\bullet]$ ', ' $\bullet$ ', ' $(\bullet)^{\mathrm{T}}$, , '*', and $(\bullet)^{\mathrm{H}}$, denote the expectation, estimation, transposed, complex conjugate, and Hermitian. We term the $i$-th column of the unit matrix $\mathbf{1}_{N} \in \mathbb{C}^{N \times N} \boldsymbol{e}_{i}^{(N)} \cdot \boldsymbol{e}^{(N)} \in \mathbb{C}^{N}$ and $\mathbf{0}_{N \times G}$ represent the $N$-dimensional column vector with one elements and the zero matrix of size $N \times G$. 


\section{CHANNEL MODEL}

We consider a time-variant frequency-selective channel impulse response (CIR)

$$
\mathrm{h}(\tau, t)=\sum_{g=0}^{G} \mathrm{a}_{g}(t) \delta\left(\tau-\tau_{g}\right)
$$

In order to simplify the description of the OFDM system model in Sec. III, we define the channel taps $\tau_{g}, g=$ $0, \ldots, G$ to be integer multiple values of the sampling time $T_{\mathrm{S}}$, i.e. $\tau_{g}=g T_{\mathrm{S}}, g=0, \ldots, G$. The power delay profile (PDP) $a_{g}, g=0, \ldots, G$ whose total power is equal to one $\sum_{g=0}^{G} a_{g}^{2}=1$ relates to $\mathrm{a}_{g}(t)$ as $\mathrm{E}\left[\left|\mathrm{a}_{g}(t)\right|^{2}\right]=a_{g}^{2}, g=$ $0, \ldots, G$. We generate the random time-variant processes $\mathrm{a}_{g}(t)$ as a wide sense stationary uncorrelated scattering (WSSUS) Rayleigh channel [12], [13] whose correlations in time direction are given by

$$
\mathrm{R}_{\mathrm{a}_{\mathrm{g}}(\mathrm{t})}(\tau)=\mathrm{E}\left[\mathrm{a}_{g}^{*}(t) \mathrm{a}_{g}(t+\tau)\right]=a_{g}^{2} \mathrm{~J}_{0}\left(2 \pi f_{\mathrm{D}, \max } \tau\right) .
$$

\section{OFDM SYSTEM MODEL}

OFDM systems summarize $K$ OFDM symbols and $N$ subcarriers to one OFDM frame [1]. We use the inverse fast Fourier transform (IFFT) and the fast Fourier transform (FFT) to get the time-domain symbols $x_{n}^{(k)}$ from the modulation symbols $X_{i}^{(k)}$ at the $i$-th subcarrier $(i=0, \ldots, N-1)$ of the $k$-th OFDM symbol $(k=0, \ldots, K-1)$ and vice versa. The $k$-th cyclic prefix $\boldsymbol{x}_{\mathrm{G}}^{(k)}=\left[x_{N-G}^{(k)}, \ldots, x_{N-1}^{(k)}\right]^{\mathrm{T}} \in$ $\mathbb{C}^{G}$ defined by the normalized guard time $G$ is inserted at the OFDM transmitter before the $k$-th time-domain symbol vector $\boldsymbol{x}_{\mathrm{S}}^{(k)}=\left[x_{0}^{(k)}, \ldots, x_{N-1}^{(k)}\right]^{\mathrm{T}} \in \mathbb{C}^{N}$ to get the $k$-th observation vector at the OFDM receiver

$$
\boldsymbol{y}_{\mathrm{S}}^{(k)}=\boldsymbol{H}_{\mathrm{S}}^{(k)}\left[\boldsymbol{x}_{\mathrm{G}}^{(k), \mathrm{T}}, \boldsymbol{x}_{\mathrm{S}}^{(k), \mathrm{T}}\right]^{\mathrm{T}}+\boldsymbol{n}_{\mathrm{S}}^{(k)}=\tilde{\boldsymbol{H}}_{\mathrm{S}, \mathrm{cyc}}^{(k)} \boldsymbol{x}_{\mathrm{S}}^{(k)}+\boldsymbol{n}_{\mathrm{S}}^{(k)}
$$

having the same structure $\boldsymbol{y}_{\mathrm{S}}^{(k)}=\left[y_{0}^{(k)}, \ldots, y_{N-1}^{(k)}\right]^{\mathrm{T}} \in \mathbb{C}^{N}$ as the symbol vector. We have $(i=0, \ldots, N-1)$

$\left(\boldsymbol{H}_{\mathrm{S}}^{(k)}\right)_{i+1, j+1}=\mathrm{a}_{G-j+i}\left((k(N+G)+i) T_{\mathrm{S}}\right), i \leq j \leq G+i$,

for the non-zero entries of the $k$-th Toeplitz channel matrix $\boldsymbol{H}_{\mathrm{S}}^{(k)} \in \mathbb{C}^{N \times(N+G)}$ according to (1). In the same way, we determine the non-zero elements $(i=0, \ldots, N-1)$

$$
\begin{aligned}
\left(\tilde{\boldsymbol{H}}_{\mathrm{S}, \mathrm{cyc}}^{(k)}\right)_{i+1, j+1}= & \mathrm{a}_{(-j+i) \bmod N}\left((k(N+G)+i) T_{\mathrm{S}}\right), \\
& (-j+i) \bmod N \leq G,
\end{aligned}
$$

of the $k$-th rearranged matrix $\tilde{\boldsymbol{H}}_{\mathrm{S}, \text { cyc }}^{(k)} \in \mathbb{C}^{N \times N}$ which becomes a real cyclic matrix for $f_{\mathrm{D}, \max }=0$. (3) incorporates the definition of the noise vector $\boldsymbol{n}_{\mathrm{S}}^{(k)}=\left[\boldsymbol{n}_{0}^{(k)}, \ldots, \boldsymbol{n}_{N-1}^{(k)}\right]^{\mathrm{T}} \in$ $\mathbb{C}^{N}$. If we transform (3) into the frequency domain, we get

$$
Y_{i}^{(k)}=H_{i, 0}^{(k)} X_{i}^{(k)}+\underbrace{\sum_{d=1}^{N-1} H_{i, d}^{(k)} X_{(i-d) \bmod N}^{(k)}}_{=I_{i}^{(k)}}+N_{i}^{(k)}
$$

$$
\begin{aligned}
& Y_{i}^{(k)}, N_{i}^{(k)}, \text { and } \\
& H_{i, d}^{(k)}=\frac{1}{N} \sum_{g=0}^{G} \sum_{n=0}^{N-1} \mathrm{a}_{g}\left((k(N+G)+n) T_{\mathrm{S}}\right) \exp \left(-\frac{\mathrm{j} 2 \pi n d}{N}\right) \\
& \exp \left(-\frac{\mathrm{j} 2 \pi g(i-d)}{N}\right)
\end{aligned}
$$

term the FFT of the observation sequence $y_{n}^{(k)}$, the noise sequence $n_{n}^{(k)}$, and the time-variant channel, respectively. The ICI $l_{i}^{(k)}$ in the $i$-th subcarrier $(i=0, \ldots, N-1)$ of the $k$-th OFDM symbol $(k=0, \ldots, K-1)$ in (6) is determined by the spectral channel components $H_{i, d}^{(k)}$ for $d=1, \ldots, N-1$. Clearly, we need both the time direction $d=0, \ldots, N-1$ and the frequency direction $d=0, \ldots, N-1$ to describe $H_{i, d}^{(k)}$ entirely. For $d=0$, $H_{i, d}^{(k)}$ states

$$
H_{i, 0}^{(k)}=\sum_{g=0}^{G} \underbrace{\frac{1}{N} \sum_{n=0}^{N-1} \mathrm{a}_{g}\left((k(N+G)+n) T_{\mathrm{S}}\right)}_{=\bar{a}_{g}^{(k)}} \exp \left(-\frac{\mathrm{j} 2 \pi g i}{N}\right) .
$$

The additional expression in (8) plays a key role for our ICI mitigation algorithms in Subsec. V-B.

\section{PILOT-AIDED CHANNEL ESTIMATION IN OFDM SYSTEMS}

In this contribution, we assume a regular placement of $L$ pilots $X_{\ell_{i}}^{(k)}=P_{\ell_{i}}, \quad \ell_{i}=i(N / L), i=0, \ldots, L-1$ for all OFDM symbols [2]. We get from (6)

$$
\hat{H}_{\ell_{i}, 0}^{(k)}=\frac{Y_{\ell_{i}}^{(k)}}{P_{\ell_{i}}}=H_{\ell_{i}, 0}^{(k)}+\frac{I_{\ell_{i}}^{(k)}+N_{\ell_{i}}^{(k)}}{P_{\ell_{\ell_{i}}}},
$$

and by applying the IFFT

$$
\hat{\bar{a}}_{g}^{(k)}=\frac{1}{L} \sum_{i=0}^{L-1} \hat{H}_{\ell_{i}, 0}^{(k)} \exp \left(\frac{\mathrm{j} 2 \pi g i}{L}\right), g=0, \ldots, G .
$$

A vanishing $f_{\mathrm{D}, \max }=0$ would induce $H_{i, d}^{(k)}=0, d=$ $1, \ldots, N-1$ and thereby $I_{i}^{(k)}=0$. For $f_{\mathrm{D}, \max }>0$, the channel estimates $\hat{H}_{\ell_{i}, 0}$ are corrupted by $\ell_{\ell_{i}}$ which motivates the design of ICI mitigation algorithms. Before executing the new ICI mitigation in Sec. V, estimates of (10) are preprocessed by a threshold operation [7]

$$
\left|\hat{\bar{a}}_{g}^{(k)}\right|<\max _{g=0, \ldots, G}\left|\hat{a}_{g}^{(k)}\right| / a_{\text {level }} \Rightarrow \hat{a}_{g}^{(k)}=0 .
$$

(11) erases those channel paths whose power is below $1 / a_{\text {level }}^{2}$ of the current maximum path power. In [7], the choice $a_{\text {level }}=10$ was shown to be a good tradeoff between efficient thresholding on the one hand and an accurate channel estimation on the other. The preprocessing of the channel estimates in (11) allows also to determine the number of nonzero channel amplitudes which have to be estimated. Apart from a reduction of the noise and interference power of $\hat{\bar{a}}_{g}^{(k)}$, the rule in (11) decreases the computational complexity of our ICI mitigation methods. 


\section{ICI MITIGATION IN OFDM SYSTEMS}

Contrary to previous publications in the area of efficient ICI mitigation, which resort to Taylor expansion [7] or to Lagrangian polynomials [8], we perform time-variant channel estimation using the Slepian functions [11]. In Subsec. VA, we summarize the theoretical background for the efficient decomposition of time-variant mobile radio channels. Subsec. V-B describes an efficient ICI mitigation approach resorting to channel estimates from adjacent OFDM symbols.

\section{A. Channel Decompositions Using Correlation Matrices} $\boldsymbol{a}_{\mathrm{G}, g}^{(k)}=\left[\mathrm{a}_{g}\left((-G+(N+G) k) T_{\mathrm{S}}\right), \ldots, \mathrm{a}_{g}((k(N+G)-\right.$ $\left.\left.1) T_{\mathrm{S}}\right)\right]^{\mathrm{T}} \in \mathbb{C}^{G}$ and $\boldsymbol{a}_{\mathrm{S}, g}^{(k)}=\left[\mathrm{a}_{g}\left((N+G) k T_{\mathrm{S}}\right), \ldots, \mathrm{a}_{g}((k(N+\right.$ $\left.\left.G)+N-1) T_{\mathrm{S}}\right)\right]^{\mathrm{T}} \in \mathbb{C}^{N}$ denote the channel vector corresponding to the $g$-th channel tap of the $k$-th guard interval and OFDM symbol, respectively. The channel sample vector of the whole OFDM symbol including the guard interval states

$$
\boldsymbol{a}_{\mathrm{GS}, g}^{(k)}=\left[\boldsymbol{a}_{\mathrm{G}, g}^{(k), \mathrm{T}}, \boldsymbol{a}_{\mathrm{S}, g}^{(k), \mathrm{T}}\right]^{\mathrm{T}} \in \mathbb{C}^{N+G},
$$

and the accumulation of $N_{\mathrm{S}}=\ell-k+1$ whole OFDM symbols belonging to the indices $k$ to $\ell$ is abbreviated by

$$
\boldsymbol{a}_{\mathrm{GS}, g}^{(k, l)}=\left[\boldsymbol{a}_{\mathrm{GS}, g}^{(k), \mathrm{T}}, \ldots, \boldsymbol{a}_{\mathrm{GS}, g}^{(l), \mathrm{T}}\right]^{\mathrm{T}} \in \mathbb{C}^{N_{\mathrm{S}}(N+G)} .
$$

The proposed decomposition of the sampled Rayleigh processes $\boldsymbol{a}_{\mathrm{GS}, g}^{(k, l)} \in \mathbb{C}^{N_{\mathrm{S}}(N+G)}$

$$
\boldsymbol{a}_{\mathrm{GS}, g}^{(k, l)}=\tilde{\boldsymbol{Q}}_{\mathrm{PC}} \tilde{\boldsymbol{Q}}_{\mathrm{PC}}^{\mathrm{H}} \boldsymbol{a}_{\mathrm{GS}, g}^{(k, l)}+\left(\mathbf{1}_{N_{\mathrm{S}}(N+G)}-\tilde{\boldsymbol{Q}}_{\mathrm{PC}} \tilde{\boldsymbol{Q}}_{\mathrm{PC}}^{\mathrm{H}}\right) \boldsymbol{a}_{\mathrm{GS}, g}^{(k, l)},
$$

where the subspace matrix $\tilde{\boldsymbol{Q}}_{\mathrm{PC}} \in \mathbb{C}^{N_{\mathrm{S}}(N+G) \times R}$ has $R \ll$ $N_{\mathrm{S}}(N+G)$ orthonormal columns. The robust computation of this subspace matrix requires the definition of the phasor vector $\boldsymbol{\varphi}_{\mathrm{S}}^{(k)}\left(f_{\mathrm{D}}\right) \in \mathbb{C}^{N}$ consisting of $\left(\boldsymbol{\varphi}_{\mathrm{S}}^{(k)}\left(f_{\mathrm{D}}\right)\right)_{n}=$ $\exp \left(\mathrm{j} 2 \pi f_{\mathrm{D}}((N+G) k+n-1) T_{\mathrm{S}}\right), \quad n=1, \ldots, N$. Likewise, we define the phasor vector for the guard interval $\boldsymbol{\varphi}_{\mathrm{G}}^{(k)}\left(f_{\mathrm{D}}\right) \in \mathbb{C}^{G}$ with the elements $\left(\boldsymbol{\varphi}_{\mathrm{G}}^{(k)}\left(f_{\mathrm{D}}\right)\right)_{g}=$ $\exp \left(\mathrm{j} 2 \pi f_{\mathrm{D}}(-G+(N+G) k+g-1) T_{\mathrm{S}}\right), g=1, \ldots, G$, and the phasor vectors corresponding to whole OFDM symbols incorporating the guard interval are

$$
\begin{gathered}
\boldsymbol{\varphi}_{\mathrm{GS}}^{(k)}\left(f_{\mathrm{D}}\right)=\left[\boldsymbol{\varphi}_{\mathrm{G}}^{(k), \mathrm{T}}\left(f_{\mathrm{D}}\right), \boldsymbol{\varphi}_{\mathrm{S}}^{(k), \mathrm{T}}\left(f_{\mathrm{D}}\right)\right]^{\mathrm{T}} \in \mathbb{C}^{N+G}, \\
\boldsymbol{\varphi}_{\mathrm{GS}}^{(k, l)}\left(f_{\mathrm{D}}\right)=\left[\boldsymbol{\varphi}_{\mathrm{GS}}^{(k), \mathrm{T}}\left(f_{\mathrm{D}}\right), \ldots, \boldsymbol{\varphi}_{\mathrm{GS}}^{(l), \mathrm{T}}\left(f_{\mathrm{D}}\right)\right]^{\mathrm{T}} \in \mathbb{C}^{N_{\mathrm{S}}(N+G)} .
\end{gathered}
$$

Therefore, we determine the optimum $\boldsymbol{Q}_{\mathrm{PC}} \in$ $\mathbb{C}^{N_{\mathrm{S}}(N+G) \times R}$ by means of the bandlimitation of $\mathrm{a}_{g}(t), g=0, \ldots, G$ to $f_{\mathrm{D}, \max }$ as

$$
\boldsymbol{Q}_{\mathrm{PC}}=\underset{\tilde{\boldsymbol{Q}}_{\mathrm{PC}}}{\operatorname{argmin} \mathrm{E}}\left[\left\|\left(\mathbf{1}_{N_{\mathrm{S}}(N+G)}-\tilde{\boldsymbol{Q}}_{\mathrm{PC}} \tilde{\boldsymbol{Q}}_{\mathrm{PC}}^{\mathrm{H}}\right) \boldsymbol{\varphi}_{\mathrm{GS}}^{(k, l)}\left(f_{\mathrm{D}}\right)\right\|_{2}^{2}\right] \text {. }
$$

$\boldsymbol{Q}_{\mathrm{PC}} \in \mathbb{C}^{N_{\mathrm{S}}(N+G) \times R}$ contains the $N_{\mathrm{PC}}=R$ eigenvectors belonging to the $N_{\mathrm{PC}}=R$ dominant eigenvalues of the autocorrelation matrix $\boldsymbol{R}_{\boldsymbol{\varphi}_{\mathrm{GS}}^{(k)}\left(f_{\mathrm{D}}\right)}=\mathrm{E}\left[\boldsymbol{\varphi}_{\mathrm{GS}}^{(k)}\left(f_{\mathrm{D}}\right) \boldsymbol{\varphi}_{\mathrm{GS}}^{(k)}\left(f_{\mathrm{D}}\right)^{\mathrm{H}}\right]$ if we assume for the random Doppler frequency $f_{\mathrm{D}}$ a uniform distribution in $\left[-f_{\mathrm{D}, \max }, f_{\mathrm{D}, \max }\right]$. This robust approach for any
TABLE I

SYSTEM PARAMETERS

\begin{tabular}{|l|r|}
\hline system parameter & value \\
\hline modulation alphabet & 8 -PSK \\
number of subcarriers & $N=892$ \\
number of pilot carriers & $L=223$ \\
number of data carriers & $N-L=669$ \\
normalized guard interval & $G=173$ \\
sampling time & $T_{\mathrm{S}}=0.26 \cdot 10^{-6} \mathrm{~s}$ \\
\hline
\end{tabular}

$\mathrm{R}_{\mathrm{ag}_{\mathrm{g}}(\mathrm{t})}(\tau)$ is necessary, since the channel correlation function in (2) cannot be assumed to be known to the receiver. As a result, we obtain $Q_{\mathrm{PC}} \in \mathbb{C}^{N_{\mathrm{S}}(N+G) \times R}$ as a discrete prolate spheriodical sequence (DPSS) expansion [10], [11].

\section{B. ICI Mitigation Using Adjacent OFDM Symbols}

In this subsection, we implement our new ICI mitigation approach resorting to the values $\hat{a}_{g}^{(k)}, g=0, \ldots, G$ given for the respective $k$-th OFDM symbol from (10) and (11). $N_{\text {PC }}$ denotes the dimension of the $g$-th coefficient vector $\boldsymbol{b}_{g} \in \mathbb{C}^{N_{\mathrm{PC}}}, g=0, \ldots, G$ and $N_{\mathrm{S}}=\ell-k+1$ gives the number of successive OFDM symbols which are used for ICI mitigation. If we compute the Slepian sequence subspace matrix $\boldsymbol{Q}_{\mathrm{PC}} \in \mathbb{C}^{N_{\mathrm{S}}(N+G) \times N_{\mathrm{PC}}}$ belonging to $N_{\mathrm{S}}=\ell-k+1$ successive OFDM symbols, we get

$$
\boldsymbol{a}_{\mathrm{GS}, g}^{(k, l)} \approx \boldsymbol{Q}_{\mathrm{PC}} \boldsymbol{b}_{g} \in \mathbb{C}^{N_{\mathrm{S}}(N+G)} \text {. }
$$

We are able to determine the unknown expansion coefficient vectors $\boldsymbol{b}_{g}, g=0, \ldots, G$ for the Slepian function subspaces by (cf. the definitions of $\bar{a}_{g}^{(k)}, \ldots, \bar{a}_{g}^{(\ell)}$ in (8))

$$
\underbrace{\left[\begin{array}{c}
\hat{\bar{a}}_{g}^{(k)} \\
\vdots \\
\hat{\bar{a}}_{g}^{(\ell)}
\end{array}\right]}_{=\hat{\bar{a}}_{g}^{(k, \ell)}}=\overline{\boldsymbol{Q}}_{\mathrm{PC}} \boldsymbol{b}_{g},
$$

if we define for $n_{\mathrm{S}}=1, \ldots, N_{\mathrm{S}}$ and $n_{\mathrm{PC}}=1, \ldots, N_{\mathrm{PC}}$

$$
\left(\overline{\boldsymbol{Q}}_{\mathrm{PC}}\right)_{n_{\mathrm{S}}, n_{\mathrm{PC}}}=\frac{1}{N} \sum_{n=G+1}^{N+G}\left(\boldsymbol{Q}_{\mathrm{PC}}\right)_{n+\left(n_{\mathrm{S}}-1\right)(N+G), n_{\mathrm{PC}}} .
$$

We get $\overline{\boldsymbol{Q}}_{\mathrm{PC}} \in \mathbb{C}^{N_{\mathrm{S}} \times N_{\mathrm{PC}}}$ beginning from $\boldsymbol{Q}_{\mathrm{PC}} \in$ $\mathbb{C}^{N_{\mathrm{S}}(N+G) \times N_{\mathrm{PC}}}$ by averaging the column entries belonging to the respective OFDM symbol without the guard interval. If the equality relationship $N_{\mathrm{S}}=N_{\mathrm{PC}}$ holds, we have

$$
\boldsymbol{b}_{g}=\overline{\boldsymbol{Q}}_{\mathrm{PC}}^{-1} \hat{\overline{\mathbf{a}}}_{g}^{(k, \ell)} \text {. }
$$

We skip the cases $N_{\mathrm{S}}>N_{\mathrm{PC}}$ and $N_{\mathrm{S}}<N_{\mathrm{PC}}$ which requires to replace $\overline{\boldsymbol{Q}}_{\mathrm{PC}}^{-1}$ in $(21)$ by $\left(\overline{\boldsymbol{Q}}_{\mathrm{PC}}^{\mathrm{H}} \overline{\boldsymbol{Q}}_{\mathrm{PC}}\right)^{-1} \overline{\boldsymbol{Q}}_{\mathrm{PC}}^{\mathrm{H}}$ or $\overline{\boldsymbol{Q}}_{\mathrm{PC}}^{\mathrm{H}}\left(\overline{\boldsymbol{Q}}_{\mathrm{PC}} \overline{\boldsymbol{Q}}_{\mathrm{PC}}^{\mathrm{H}}\right)^{-1}$, respectively, due to space limitations.

\section{ICI POWER COMPUTATION}

Before we investigate the bit error ratio (BER) performance of our ICI cancellation methods, we show how our ICI mitigation methods improve the signal to interference ratio 
(SIR) at the receiver. Tab. I summarizes the most important simulation parameters [7]. This SIR analysis considers a narrowband channel $\left(a_{0}=1\right.$ and $\left.a_{g}=0, g=1, \ldots, G\right)$. We obtain as average SIR $\xi$ for any OFDM symbol [7]

$$
\begin{aligned}
\xi & =\frac{N}{\sum_{n=0}^{N-1} \mathrm{E}\left[\left|\frac{\mathrm{a}_{0}\left(n T_{\mathrm{S}}\right)-\hat{\mathrm{a}}_{0}\left(n T_{\mathrm{S}}\right)}{\mathrm{a}_{0}\left(n T_{\mathrm{S}}\right)}\right|^{2}\right]} \\
& \approx \frac{N}{\sum_{n=0}^{N-1} \frac{\mathrm{E}\left[\left|\mathrm{e}_{0}\left(n T_{\mathrm{S}}\right)\right|^{2}\right]\left(\rho_{0}\left(n T_{\mathrm{S}}\right)^{2}+\operatorname{Ei}(-\ln (1-\varepsilon))\left(1-\rho_{0}\left(n T_{\mathrm{S}}\right)^{2}\right)\right)}{\mathrm{E}\left[\left|\hat{\mathrm{a}}_{0}\left(n T_{\mathrm{S}}\right)\right|^{2}\right]}},
\end{aligned}
$$

where $\operatorname{Ei}(\bullet)$ and $\ln (\bullet)$ denote the exponential integral and the natural logarithm. In (22), we use the estimation error

$$
\mathrm{e}_{0}\left(n T_{\mathrm{S}}\right)=\mathrm{a}_{0}\left(n T_{\mathrm{S}}\right)-\hat{\mathrm{a}}_{0}\left(n T_{\mathrm{S}}\right)
$$

as well as the normalized correlation coefficient

$$
\rho_{0}\left(n T_{\mathrm{S}}\right)=\frac{\mathrm{E}\left[\mathrm{e}_{0}\left(n T_{\mathrm{S}}\right) \hat{\mathrm{a}}_{0}\left(n T_{\mathrm{S}}\right)^{*}\right]}{\sqrt{\mathrm{E}\left[\left|\mathrm{e}_{0}\left(n T_{\mathrm{S}}\right)\right|^{2}\right]} \sqrt{\mathrm{E}\left[\left|\hat{\mathrm{a}}_{0}\left(n T_{\mathrm{S}}\right)\right|^{2}\right]}}
$$

between the estimation error $e_{0}\left(n T_{\mathrm{S}}\right)$ and the channel estimate $\hat{a}_{0}\left(n T_{\mathrm{S}}\right)$. The analytical SIR computation We use the Rayleigh PDF assumption to get meaningful SIR values which exclude for $\varepsilon=10^{-6}$ the estimates $\hat{a}_{0}\left(n T_{\mathrm{S}}\right)$ below the threshold $\left|\hat{\mathrm{a}}_{0}\left(n T_{\mathrm{S}}\right)\right|^{2}<\varepsilon \mathrm{E}\left[\left|\hat{\mathrm{a}}_{0}\left(n T_{\mathrm{S}}\right)\right|^{2}\right]$. In order to evaluate (22), we set $\hat{a}_{0}\left(n T_{\mathrm{S}}\right)=\hat{a}_{0}^{(0)}, n=0, \ldots, N-1$ or

$$
\hat{\mathbf{a}}_{\mathrm{S}, 0}^{(0)}=\hat{a}_{0}^{(0)} \boldsymbol{e}^{(N)}
$$

in the case of no ICI mitigation. For our ICI mitigation algorithm with $N_{\mathrm{S}}=N_{\mathrm{PC}}$, we get

$$
\hat{\boldsymbol{a}}_{\mathrm{S}, 0}^{(0)}=\left(\boldsymbol{e}_{1}^{\left(N_{\mathrm{S}}\right), \mathrm{T}}\left[\mathbf{0}_{N \times G}, \mathbf{1}_{N}\right]\right) \boldsymbol{Q}_{\mathrm{PC}} \overline{\boldsymbol{Q}}_{\mathrm{PC}}^{-1} \hat{\mathbf{a}}_{0}^{\left(0, N_{\mathrm{S}}-1\right)} .
$$

We refer for $\mathrm{E}\left[\left|\hat{\mathrm{a}}_{0}\left(n T_{\mathrm{S}}\right)\right|^{2}\right], \mathrm{E}\left[\left|\mathrm{e}_{0}\left(n T_{\mathrm{S}}\right)\right|^{2}\right]$, and $\rho_{0}\left(n T_{\mathrm{S}}\right)$ for the two considered cases in (25) and (26) to [7].

The results in Fig. 1 for no ICI mitigation and $N_{\mathrm{S}}=$ $N_{\text {PC }}=1$ are the same due to the SIR denominator in (22) and (25) and (26). We see that the elements of $\hat{a}_{\mathrm{S}, 0}^{(0)}$ in (25) and (26) become the same apart from a constant multiplier. Hence the SIR $\xi$ remains unchanged, and for this reason we skip in Sec. VII the BER simulations of $N_{\mathrm{S}}=N_{\mathrm{PC}}=1$. However, we see a considerable SIR improvement, if we resort to the Slepian subspace based ICI mitigation for $N_{\mathrm{S}}=N_{\mathrm{PC}}>1$. The comparison of different subspace dimensions $N_{\mathrm{S}}=N_{\mathrm{PC}}=2, \ldots, 5$ yields that the optimum SIR is obtained for an increased maximum Doppler frequency also for growing $N_{\mathrm{S}}=N_{\mathrm{PC}}$. The SIR comparison with [7] leads to an improvement especially for large $f_{\mathrm{D}, \max }$ and growing $N_{\mathrm{S}}=N_{\mathrm{PC}}$.

\section{Simulations AND Results}

We consider the two testing PDPs from [7] in order to compare our new ICI mitigation algorithms to the previous results. The first of the two examples termed PDP 1 contains only two non-zero amplitudes $a_{g}, g=0, \ldots, G$

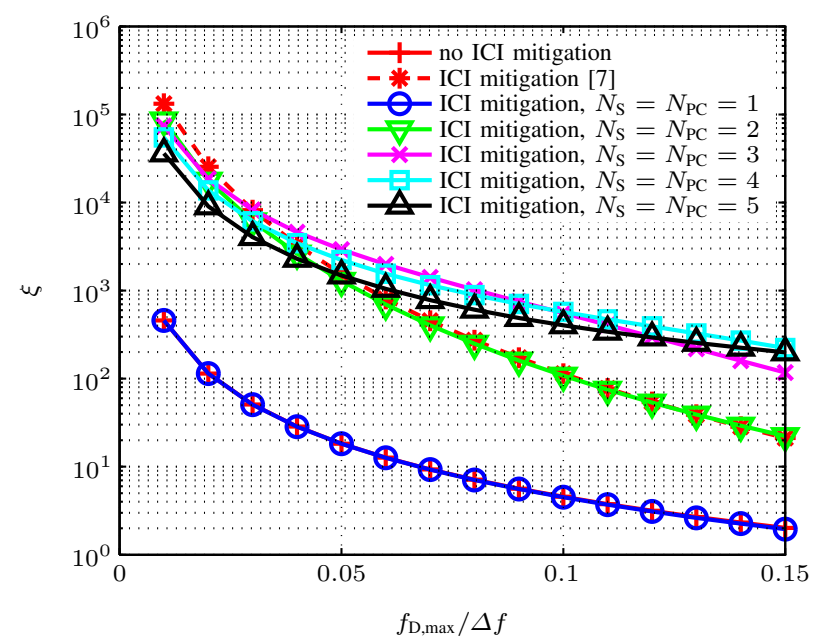

Fig. 1. Analytical SIR $\xi$ versus the normalized maximum Doppler frequency $f_{\mathrm{D} \text {,max }}$ without and with Slepian subspace based ICI mitigation.

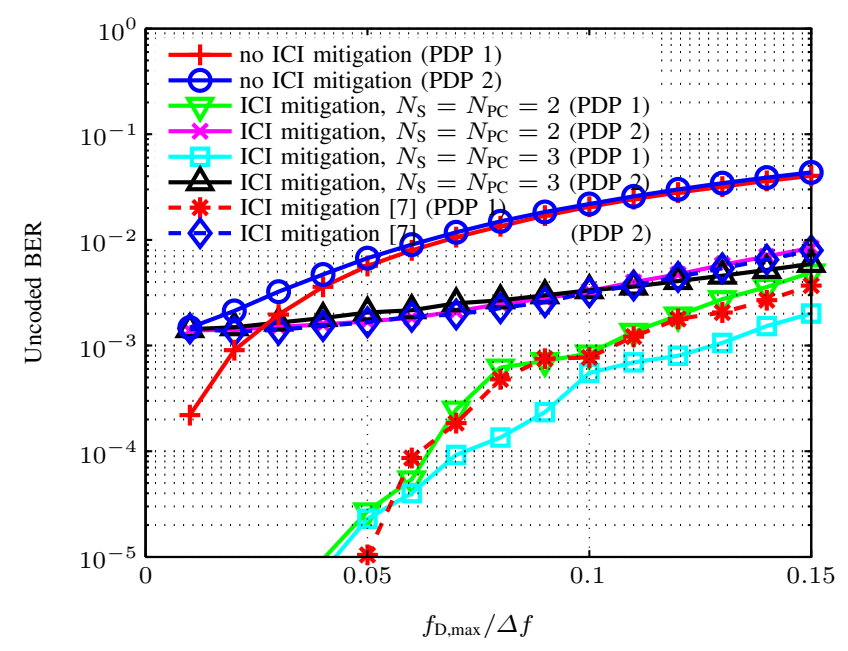

Fig. 2. Uncoded BERs versus the normalized maximum Doppler frequency $f_{\mathrm{D}, \max }$ with and without ICI mitigation based on Slepian functions.

for $g=0$ and $g=77$. We have $a_{0}=a_{77}=$ $1 / \sqrt{2}$ due to the normalization of the channel power. PDP 2 is endowed with a larger number of non-zero channel amplitudes for $g=0,1,2,4,5,6,8,9,12$ and $g=$ $130,131,132,134,135,136,138,139,142$ with powers $a_{0}^{2}=$ $a_{130}^{2}=0.06, a_{1}^{2}=a_{131}^{2}=0.1, a_{2}^{2}=a_{132}^{2}=0.05, a_{4}^{2}=$ $a_{134}^{2}=0.04, a_{5}^{2}=a_{135}^{2}=0.06, a_{6}^{2}=a_{136}^{2}=0.095, a_{8}^{2}=$ $a_{138}^{2}=0.05, \quad a_{9}^{2}=a_{139}^{2}=0.035, a_{12}^{2}=a_{142}^{2}=0.01$. Both PDPs describe the typical channel reflections in single frequency networks (SFNs) when the mobile station (MS) is closely located to one of the base stations (BSs).

Fig. 2 summarizes the simulations for PDP 1 and 2 for different maximum Doppler frequencies $f_{\mathrm{D}, \max }$ normalized to the subcarrier spacing $\Delta f$. This first simulation studies the interference effects caused by the Doppler or the delay spread. If we consider the BER performance of the OFDM 


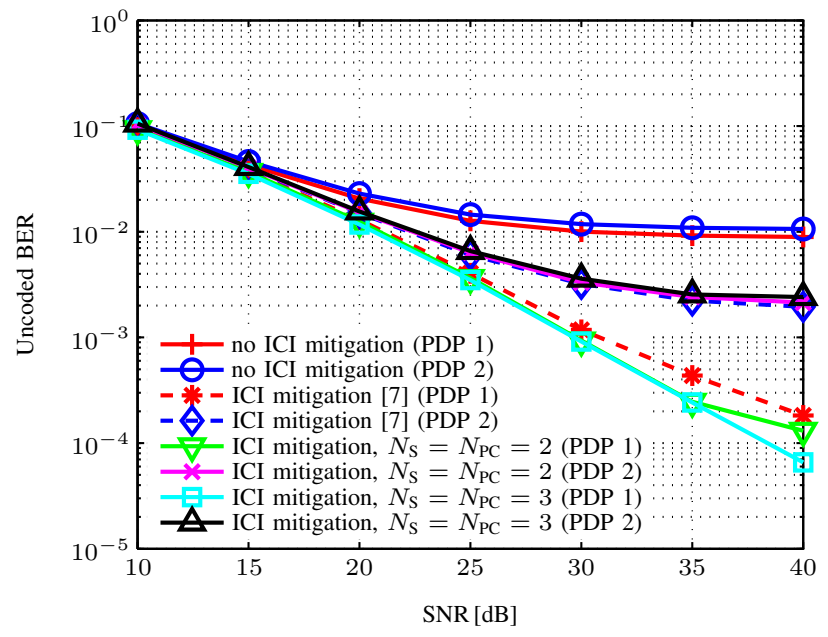

Fig. 3. Uncoded BERs versus the SNR with and without ICI mitigation based on Slepian functions for $f_{\mathrm{D}, \max } / \Delta f=0.065$.

system without any ICI mitigation, we observe a smaller BER for PDP 1 compared to PDP 2 because of the smaller delay spread. This BER difference vanishes for an increasing Doppler spread, since the ICI becomes the dominant interference. If we look at the BER performance of the Slepian subspace based ICI mitigation, we observe for both PDPs a substantial BER reduction. We see a substantial BER improvement for PDP 2 which increases for a growing maximum Doppler frequency $f_{\mathrm{D}, \max }$. The Slepian subspace based ICI mitigation for $N_{\mathrm{S}}=N_{\mathrm{PC}}=2$ and $N_{\mathrm{S}}=N_{\mathrm{PC}}=3$ yields approximately the same BER performance for PDP 2. However, an increased subspace dimension $N_{\mathrm{S}}=N_{\mathrm{PC}}$ enables a further BER reduction if we consider PDP 1, which is also due to the smaller delay spread. Likewise as for PDP 2, for a growing Doppler spread the ICI becomes the prevalent interference and is decreased efficiently by our ICI mitigation algorithm. It is also important to mention that the SIR in Fig. 1 maps onto the BERs of both PDPs, since the optimum $N_{\mathrm{S}}=N_{\mathrm{PC}}$ dependent on $f_{\mathrm{D}, \max }$ for a maximum SIR in Fig. 1 and a minimum BER in Fig. 2 is identical. Looking at the results from [7], we see an additional BER gain of the Slepian subspace based ICI mitigation.

In Fig. 3, we consider the BER performance for $f_{\mathrm{D}, \max } / \Delta f=0.065$ and different signal to noise ratios (SNRs). Within the SNR range between $10 \mathrm{~dB}$ and $15 \mathrm{~dB}$ ), the BER performance cannot be improved by ICI mitigation since the dominant BER source is the noise. However, for high SNR values, the improvements of Slepian subspace based ICI mitigation become apparent. The curves which skip the ICI mitigation methods saturate at a BER of approximately $10^{-2}$. Likewise as we already observed for Fig. 2, the performance gain of ICI mitigation for PDP 1 is larger due to the smaller delay spread. Fig. 3 reveals likewise as Fig. 2 that different subspace dimensions $N_{\mathrm{S}}=N_{\mathrm{PC}}$ pose a further possibility to decrease the ICI. The comparison of the BER values at high SNRs in Fig. 3 and [7] yields that our algorithms outperform the Taylor expansion based ICI mitigation. Especially the BERs for PDP 1 show the superiority of our novel ICI mitigation. The corresponding BERs with $N_{\mathrm{S}}=N_{\mathrm{PC}}=2$ and $N_{\mathrm{S}}=N_{\mathrm{PC}}=3$ are remarkably smaller than the result from [7].

\section{CONCLUSIONS}

This contribution deals with new algorithms for the ICI mitigation in OFDM systems with high mobility. Beginning with pilot-aided channel estimation for a given OFDM frame, we analyze the influence of ICI onto the channel estimations for the pilot subcarriers. Simulations prove that their direct use for data detection yields a poor transmission quality. The key idea of the new ICI mitigation method consists in the determination of the unknown basis expansion coefficients using channel estimates from adjacent OFDM symbols. This procedure enables a data detection, which mitigates the ICI almost entirely even for high mobility OFDM systems.

\section{ACKNOWLEDGEMENT}

The research leading to these results has received funding from the European Union's Seventh Framework Programme (FP7/2007-2013) under grant agreement $\mathrm{n}^{\circ} 227890$ (GRAMMAR project).

\section{REFERENCES}

[1] ETSI EN 300744 V1.5.1, European Standard (Telecommunications Series) Digital Video Broadcasting (DVB); Framing Structure, Channel Coding and Modulation for Digital Terrestrial Television, November 2004.

[2] ETSI TS 101475 V1.3.1, Technical Specification Broadband Radio Access Networks (BRAN); HIPERLAN Type 2; Physical (PHY) Layer, December 2001.

[3] IEEE Std 802.11a-Part 11 : Wireless LAN Medium Access Control (MAC) and Physical Layer (PHY) Specifications High-Speed Physical Layer in the $5 \mathrm{GHz}$ Band, November 1999.

[4] M. Russell and G. Stuber, "Interchannel Interference Analysis of OFDM in a Mobile Environment," in Proceedings of the Semiannual IEEE International Conference on Vehicular Technology (VTC Fall 1995), September 1995.

[5] M. Schellmann, "Suppressing Doppler-induced Intercarrier Interference with Multiple Receiving Antennas," in Proceedings of the International OFDM Workshop (InOWO 2008), Hamburg, Germany, August 2008.

[6] W. G. Jeon, K. H. Chang, and Y. S. Cho, "An Equalization Technique for Orthogonal Frequency-Division Multiplexing Systems in TimeVariant Multipath Channels," IEEE Transactions on Communications, vol. 47, no. 1, pp. 27-32, January 1999.

[7] Y. Mostofi and D. C. Cox, "ICI Mitigation for Pilot-Aided OFDM Mobile Systems," IEEE Transactions on Wireless Communications, vol. 4, no. 2, pp. 765-774, March 2005.

[8] A. R. Ali, T. J. Khanzada, and A. Omar, "ICI Cancellation for OFDM Systems Using Lagrange Polynomial Approximation," in Proceedings of the International OFDM Workshop (InOWO 2008), Hamburg, Germany, August 2008.

[9] R. Negi and J. Cioffi, "Pilottone Selection for Channel Estimation in a Mobile OFDM System," IEEE Transactions on Consumer Electronics, vol. 44, no. 3, pp. 1122-1128, August 1998.

[10] H. L. van Trees, Optimum Array Processing, John Wiley, New York, USA, 2002.

[11] T. Zemen and C. F. Mecklenbräuker, "Time-Variant Channel Estimation Using Discrete Prolate Spheroidal Sequences," IEEE Transactions on Signal Processing, vol. 53, no. 9, pp. 3597-3607, September 2005.

[12] W. C. Jakes, Microwave Mobile Communications, John Wiley, IEEE Press, New York, USA, 1994.

[13] H. Meyr, M. Moeneclaey, and S. A. Fechtel, Digital Communication Receivers, John Wiley, New York, USA, 1997. 